\title{
Mental health of mothers and their premature infants for the prevention of child abuse and maltreatment
}

\author{
Yuko Ishizaki $^{{ }^{*}}$, Teruyo Nagahama ${ }^{2}$, Kazunari Kaneko ${ }^{1}$ \\ ${ }^{1}$ Department of Pediatrics, Kansai Medical University, Osaka, Japan; *Corresponding Author: ishizaky@takii.kmu.ac.jp \\ ${ }^{2}$ Graduate School of Human Life Science, Osaka City University, Osaka, Japan
}

Received 22 January 2013; revised 24 February 2013; accepted 3 March 2013

\begin{abstract}
Birth of preterm infants is a stressful event for their parents, particularly for mothers. The mothers of preterm infants often feel hard to relate their infants because they have separated since their first days after delivery. Long term separation and less attractive, less responsive appearance of preterm infants also make it difficult to build mother-child relationships. In addition, the mothers of preterm infants are likely to have mood disorders such as depression, anxiety, and stress-related disorders. The mothers' psychiatric illnesses affect the psychosocial development of preterm infants and are often regarded as a risk factor for child abuse and maltreatment in later life. Child abuse and maltreatment are also prevalent among preterm infants than the full term infants. Intervention from the early period of preterm birth is an important issue for both preterm infants and their mothers. Medical and co-medical professionals should pay attention to developmental outcome of preterm as well as psychosocial conditions of their mothers for the improvement of their mental health.
\end{abstract}

Keywords: Preterm Infants; Neonatal Intensive Care Unit; Maternal Depression; Bonding; Child Abuse

\section{INTRODUCTION}

Advances in neonatal medicine have improved survival rates and enabled the intact survival of preterm infants, even of extremely low birth weight infants (birth weight $<1000$ g). However, many potential risk factors are associated with further development of preterm infants, e.g., periventricular hemorrhage, ventricular dilation, periventricular leukomalacia, chronic neonatal lung diseases, history of infection, male gender, and being small for gestational age [1-4]. In addition to physical sequelae, emotional problems of their mothers and quality of parenting behavior may also affect the psychosocial and cognitive development of preterm infants [5-10]. With improved survival chances of preterm infants, there is growing concern for their developmental outcome and future quality of life.

The increase in survival rate of preterm infants raised the mental health problems of the infants and their mothers which have not been experienced before. That is the psychological interaction between survived infants grows with the problem of developmental deviation or delay and their mothers who often becomes depressed by blaming herself for not having given birth to a healthy baby. Therefore, to describe the situation of preterm infants and their mothers in early period after delivery and to discuss the early intervention to prevent or improve mood disturbances of the mothers appear to be important in terms of maternal and child mental health.

\section{IMPORTANCE OF MENTAL HEALTH OF MOTHERS AND THEIR PRETERM INFANTS}

Preterm infants or those with severe physical problems are obliged to spend the postnatal period in an incubator for various medical reasons and are thus separated from their mothers for a prolonged period of time. Medical care for premature infants naturally has its main focus on the infant's physical health, as provided by the neonatal intensive care unit (NICU). For parents of premature infants, not only psychological damage due to preterm delivery but also the NICU environment appears to be stressful with bright lights, noisy life support and monitoring equipment, and chemical scents [11]. Furthermore, viewing their ill infant connected to equipment by tubes and wires and surrounded by medical personnel can be very disturbing [11].

However, the greatest source of stress experienced by these parents is often the loss of their expected and desired parental role. Emotional reactions of parents to the NICU experience can vary from disappointment, guilt, 
sadness, depression, hostility, anger, fear, anxiety, grief, and loss of self-esteem [12-15]. After the birth of a premature infant, high levels of depression and anxiety are common in both parents $[13,16]$. In particular, for mothers, the experience of preterm birth is regarded as a highly stressful event, and they may find it more difficult to establish relationships with their babies after being separated from them during the initial days following delivery [17]. They tend to blame themselves for not having given birth to a healthy baby and self reproach that they caused their infants to go through painful treatment and for being unable to do anything for the child [18].

Even 1 month after birth, mothers of premature infants have been found to be at a greater risk of psychological stress than mothers of full-term infants, with $10 \%$ of the former experiencing severe symptoms of psychological distress neonatally and one-third experiencing clinical levels of depression and anxiety [15]. We and others have further confirmed that an admission of infants to NICU increased the prevalence of mood disturbance in their mothers $[19,20]$. Furthermore, the mothers of infants in NICU show increased symptoms of acute traumatic stress and depression [21,22]. It has been suggested that a traumatic maternal experience related to premature birth may have a lasting influence on motherchild interactional behavior [23,24].

\section{MATERNAL PSYCHIATRIC ILLNESS, PRETERM BIRTH, AND CHILD ABUSE}

Maternal depression, anxiety or other psychiatric illness, history of child abuse and mothers' socioeconomic disadvantages are regarded as risk factors for child abuse and maltreatment [25-28]. Previous studies have consistently found a high incidence of child abuse associated with a history of neonatal medical problems, premature birth, and low birth weight. About the relation between a premature infants and a child abuse, it goes back to prospective study in 1970's. Hunter et al. [29] reported the high incidence of maltreatment in the preterm and ill newborns and showed significant association between social isolation, a family history of child abuse and neglect, serious marital problems, inadequate child care arrangements, apathetic and dependent personality styles, and inadequate child spacing with later maltreatment. Bugental et al. [26] suggested that child maltreatment during the 1st year of life was predicted by neonatal status, such as low Apgar scores, preterm status.

Mothers delivering preterm infants are likely to have had physical or psychosocial vulnerabilities before their pregnancy. Lower socioeconomic status, poverty, minority status, substance abuse, psychiatric illness, history of child abuse, or various physical comorbidities exist in expectant mothers of preterm infants before and during pregnancy and continue to exist after birth. These unresolved problems often persist and affect parenting behavior; child abuse and maltreatment are serious problems in preterm infants.

Another explanation for the higher prevalence of child abuse and maltreatment in preterm infants involves the relationships between neonatal problems and adverse parenting behavior, causing possible delay or disturbance in the bonding process between the parent and infant [24]. According to Tooten et al. [11], "at the appearance of preterm infants is judged as less attractive than full term infants and their behavior is observed as less alert, less attentive, less active and less responsive than that of full-term infants. Furthermore, preterm infants engage in fewer broad smiles, are relatively fussy and irritable, are more difficult to soothe, show more mixed behavioral cues, show more sensory-defensive behaviors and are described as more temperamentally difficult than term peers". It is established that mothers find it more difficult to relate to infants from they have been separated during their first days following delivery. Size, appearance and responsiveness may also influence the mother's first feeling about the infants $[19,30]$.

Parental "bonding" can be described as "the establishment of an emotional connection of the parent to the infant [31]". The process of forming a bond with an infant begins during pregnancy and develops further after birth, and the process of bonding, in turn, sets the stage for the evolvement of attachment, which develops later in childhood and can be described as "the capacity to form selective, enduring and mutual relationships [11].” In case of premature birth, the process of bonding may be disturbed because the mother is often separated from the infant while medical treatment is provided. Negative parental feelings may impede the establishment of a wellbalanced parent-infant relationship and can be the source of parent-infant attachment difficulties [18]. Both maternal emotional illness and the lower sensitivity of infants may disturb healthy bonding and attachment between mothers and their infants.

\section{EARLY INTERVENTION IN REGARD TO MENTAL HEALTH OF MOTHERS OF PRETERM INFANTS}

Because both preterm birth itself and adverse parenting behavior due to mothers' psychosocial stress may increase the risk of child abuse and maltreatment in preterm infants, early intervention to reduce maternal psychosocial burden would improve child health. Intervention programs for mothers of preterm infants include psychological support, health check-ups for the child, and stabilization of mother-infant relationships $[13,14$, 
31-35]. These trials have continued through admission of infants to the NICU, discharge, and return home [36].

As a support measure to establish bonding during hospitalization of infants, skin-to-skin contact [37] such as kangaroo care [38] and touch therapy [39] contribute to the development of a healthy mother-infant relationship. Since a Colombian pediatrician concerned with the problems arising from a shortage in incubators and impact of separating women from their infants in NICU developed Kangaroo care [40], Kangaroo care is widely considered to be the most feasible, readily available, and preferred intervention for decreasing neonatal morbidity and mortality in developing countries. This method aims to restore the close relationship between the newborn and parents by placing the infant in direct skin-to-skin contact with one of them, ensuring physiological and psychological warmth and bonding. The kangaroo position provides ready access to nourishment; while the parent's stable body temperature helps regulate the neonate's temperature more smoothly than an incubator and enables readily accessible breastfeeding [40]. In the case of preterm infants, this technique not only stabilizes physical condition and breast feeding but also reduces infants' pain perception and stabilizes mothers' mood status [4147].

Touch therapy [39], another type of skin-to-skin contact, is used to heal the infant, where the mother's hands are the instrument. It is reported that touch therapy is effective in stabilizing physical status, promoting gain in body weight, and shortening admission reducing stress behavior in preterm infants treated in the NICU $[48,49]$. Both Kangaroo care and touch therapy are only feasible after the medical condition of the infants is no longer life-threatening. In case of ELBWIs in incubator, a preparatory process before touch therapies is applied that the mother gently cradle their child's head and hips, which is termed "preliminary holding". The implementation of preliminary holding helps mothers become familiar with their infants, reduce distress, and grow into motherhood [18]. Skin-to-skin contact such as Kangaroo care, touch therapy and preliminary holding are applicable to infants in the NICU, and these enhance the establishment of a healthy mother-child relationship in the early period after the birth of a preterm infant.

After discharge of an infant from the NICU, various levels of child support are available, such as peer groups, individual counseling, and home visits. Early individualized, family-based interventions during neonatal hospitalization and the transition to home have been shown to reduce parental stress and depression, increase parental self-esteem, and improve positive early parent-preterm infant interactions [50]. Ravn et al. [51] reported early interaction-based interventions on parenting and infant communication skills reduced postpartum depression and extended the period of breastfeeding. Preyde a et al. [14] suggested that parent-to-parent peer support for mothers of very preterm infants in a NICU effective in helping mothers deal with the stress of very preterm birth. During hospitalization, parental self-confidence has to be repetitively reinforced and evaluated before discharge because insecure parents at discharge are more likely to have problems with their infants at home, which may lead to persistent parent-infant relationship problems [11].

Furthermore, as a new trial, the usefulness of "prenatal visiting" is also reported [52]. Before delivery, expectant mothers of premature infants often have psychological problems such as feelings of anxiety or depression because they are afraid of miscarriage, stillbirth, or preterm birth. Admission of newborn infants to the NICU may also increase the prevalence of mood disturbance in their mothers, who then experience preterm birth as a highly stressful event. The implementations of "prenatal visiting" help mothers relax, become familiar with their infants, reduce their sense of guilt, and increase confidence in their motherhood skills and their ability to take care of their infant. These may also improve mother-child communication and interaction and yield positive consequences for the families of preterm infants in their future life together.

\section{SUMMARY}

Birth of preterm infants is very stressful for the mother and often causes psychiatric disorders. Early intervention is essential for improvement of the mother's mental health, establishment of mother-child relationships, cognitive development of the child, and prevention of child abuse and maltreatment.

\section{REFERENCES}

[1] Waugh, J., O’Callaghan, M.J., Tudehope, D.I., Mohay, H.A., Burns, Y.R., Gray, P.H. and Rogers, Y.M. (1996) Prevalence and aetiology of neurological impairment in extremely low birthweight infants. Journal of Paediatrics and Child Health, 32, 120-124. doi:10.1111/j.1440-1754.1996.tb00907.x

[2] Fawer, C.L., Besnier, S., Forcada, M., Buclin, T. and Calame, A. (1995) Influence of perinatal, developmental and environmental factors on cognitive abilities of preterm children without major impairments at 5 years. Early $\mathrm{Hu}$ man Development, 43, 151-164. doi:10.1016/0378-3782(95)01673-2

[3] Tough, S.C., Siever, J.E., Leewm, S., Johnston, D.W., Benzies, K. and Clark, D. (2008) Maternal mental health predicts risk of developmental problems at 3 years of age: Follow up of a community based trial. BMC Pregnancy and Childbirth, 8, 16. doi:10.1186/1471-2393-8-16

[4] Hutton, J.L., Pharoah, P.O., Cooke, R.W. and Stevenson, 
R.C. (1997) Differential effects of preterm birth and small gestational age on cognitive and motor development. Archives of Disease in Childhood. Fetal and Neonatal Edition, 76, F75-F81. doi:10.1136/fn.76.2.F75

[5] Grace, S.L., Evindar, A. and Stewart, D.E. (2003) The effect of postpartum depression on child cognitive development and behavior: a review and critical analysis of the literature. Archives of Women's Mental Health, 6, 263274. doi:10.1007/s00737-003-0024-6

[6] Gutteling, B.M., de Weerth, C., Zandbelt, N., Mulder, E.J., Visser, G.H. and Buitelaar, J.K. (2006) Does maternal prenatal stress adversely affect the child's learning and memory at age six? Journal of Abnormal Child Psychology, 34, 789-798. doi:10.1007/s10802-006-9054-7

[7] Heneghan, A.M., Silver, E.J., Bauman, L.J., Westbrook, L.E. and Stein, R.E. (1998) Depressive symptoms in inner-city mothers of young children: Who is at risk? Pediatrics, 102, 1394-1400. doi:10.1542/peds.102.6.1394

[8] Sinclair, D. and Murray, L. (1998) Effects of postnatal depression on children's adjustment to school. Teacher's reports. The British Journal of Psychiatry, 172, 58-63. doi:10.1192/bjp.172.1.58

[9] Wrate, R.M., Rooney, A.C., Thomas, P.F. and Cox, J.L. (1985) Postnatal depression and child development. A three-year follow-up study. The British Journal of Psychiatry, 146, 622-627. doi:10.1192/bjp.146.6.622

[10] Caplan, H.L., Cogill, S.R., Alexandra, H., Robson, K.M., Katz, R. and Kumar, R. (1989) Maternal depression and the emotional development of the child. The British Journal of Psychiatry, 154, 818-822. doi:10.1192/bjp.154.6.818

[11] Tooten, A., Hoffenkamp, H.N., Hall, R.A., Winkel, F.W., Eliëns, M., Vingerhoets, A.J. and van Bakel, H.J. (2012) The effectiveness of video interaction guidance in parents of premature infants: A multicenter randomised controlled trial. BMC Pediatrics, 12, 76. doi:10.1186/1471-2431-12-76

[12] Miles, M.S. and Holdtich-Davis, D. (1997) Parenting the prematurely born child: Pathways of influence. Seminars in Perinatology, 21, 254-266. doi:10.1016/S0146-0005(97)80067-5

[13] Meyer, E.C., Coll, C.T.G., Seifer, R., Ramos, A., Kilis, E. and Oh, W. (1995) Psychological distress in mothers of preterm infants. Developmental and behavior Pediatrics, 16, 412-417.

[14] Preyde, M. and Ardal F. (2003) Effectiveness of a parent "buddy" program for mothers of very preterm infants in a neonatal intensive care unit. Canadian Medical Association Journal, 168, 969-973.

[15] Singer, L.T., Salvator, A., Guo, S., Collin, M., Lilien, L. and Baley, J. (1999) Maternal psychological distress and parenting stress after the birth of a very low-birth-weight infant. The Journal of the American Medical Association, 281, 799-805. doi:10.1001/jama.281.9.799

[16] Miles, M.S., Funk, S.G. and Kaspar, M.A. (1992) The stress response of mothers and fathers of preterm infants. Research in Nursing \& Health, 15, 261-269. doi:10.1002/nur.4770150405

[17] Bryan, E. (2003) The impact of multiple preterm births on the family. BJOG, 110, 24-28.

[18] Ishizaki, Y., Nagahama, T., Kitamura, N., Suzuki, S., Fujii, T., Otsuka, I., Iba, M., Sakurai, C., Enomoto, Y. and Kobayashi, Y. (2007) Effects of "preliminary holding” of extremely low birth weight infants on the mental health of their mothers. International Journal of Psychology Research, 1, 23-32.

[19] Nagahama, T. and Matsushima, K. (2004) A longitudinal study of mood state of mothers of infants who were admitted to a neonatal intensive care unit. Journal of Child Health, 63, 640-646. (in Japanese)

[20] MacKey, M.C., Williams, C.A. and Tiller, C.M. (2000) Stress, pre-term labour and birth outcomes. Journal of Advanced Nursing, 32, 666-674. doi:10.1046/j.1365-2648.2000.01526.x

[21] Vanderbilt, D., Bushley, T., Young, R. and Frank, D.A. (2009) Acute posttraumatic stress symptoms among urban mothers with newborns in the neonatal intensive care unit: A preliminary study. Journal of Developmental \& Behavioral Pediatrics, 30, 50-56. doi:10.1097/DBP.0b013e318196b0de

[22] Eriksson, B.S. and Pehrsson, G. (2002) Evaluation of psycho-social support to parents with an infant born preterm. Journal of Child Health Care, 6, 19-33. doi: $10.1177 / 136749350200600103$

[23] Teti, D.M. and Gelfand, D.M. (1991) Behavioral competence among mothers of infants in the first year: The mediational role of maternal self-efficacy. Child Development, 62, 918-929. doi:10.2307/1131143

[24] Muller-Nix, C., Forcada-Guex, M., Pierrehumbert, B., Jaunin, L., Borghini, A. and Ansermet, F. (2004) Prematurity, maternal stress and mother-child interactions. Early Human Development, 79, 145-158. doi:10.1016/j.earlhumdev.2004.05.002

[25] Siefert, K., Thompson, T., ten Bensel, R.W. and Hunt, C. (1983) Perinatal stress: A study of factors linked to the risk of parenting problems. Health \& Social Work, 8, 107-121.

[26] Bugental, D.B. and Happaney, K. (2004) Predicting infant maltreatment in low-income families: the interactive effects of maternal attributions and child status at birth. Developmental Psychology, 40, 234-243. doi:10.1037/0012-1649.40.2.234

[27] Mizik, M., Bocknek, E.L., Broderick, A., Richardson, P, Rosenblum, K.L., Thelen, K. and Seng, J.S. (2013) Motherinfant bonding impairment across the first 6 months postpartum: The primacy of psychopathology in women with childhood abuse and neglect histories. Archives of Women's Mental Health, 16, 29-38. doi:10.1007/s00737-012-0312-0

[28] Strathearn, L., Gray, P.H., O’Callaghan, M.J. and Wood, D.O. (2001) Childhood neglect and cognitive development in extremely low birth infants: A prospective study. Pediatrics, 108, 142-151. doi:10.1542/peds.108.1.142

[29] Hunter, R.S., Kilstrom, N., Kraybill, E.N. and Loda, F. (1978) Antecedents of child abuse and neglect in premature infants: A prospective study in a newborn intensive care unit. Pediatrics, 61, 629-635.

[30] Goldberg, S., Perrotta, M., Minde, K. and Corter, C. (1986) 
Maternal behavior and attachment in low-birth-weight twins and singletons. Child Development, 57, 34-46. doi:10.2307/1130635

[31] Bracht, M., Ardal, F., Bot, A. and Cheng, C.M. (1998) Initiation and maintenance of a hospital-based parent group for parents of premature infants: Key factors for success. Neonatal Network, 17, 33-37.

[32] Minde, K., Shosenberg, N., Marton, P., Thompson, J., Ripley, J. and Burns, S. (1980) Self-help groups in a premature nursery-A controlled evaluation. The Journal of Pediatrics, 96, 933-940. doi:10.1016/S0022-3476(80)80586-5

[33] Landry, S.H., Smith, K.E., Miller-Loncar, C.L. and Swank, P.R. (1998) The relation of change in maternal interactive styles to the developing social competence of full-term and preterm children. Child Development, 69, 105-123.

[34] Smith, K.E., Landry, S.H., Swank, P.R., Baldwin, C.D., Denson, S.E. and Wildin, S. (1996) The relation of medical risk and maternal stimulation with preterm infants' development of cognitive, language and daily living skills. Journal of Child Psychology and Psychiatry, 37, 855-864. doi:10.1111/j.1469-7610.1996.tb01481.x

[35] Meijssen, D., Wolf, M.J., van Bakel, H., Koldewijn, K., Kok, J. and van Baar, A. (2011) Maternal attachment representations after very preterm birth and the effect of early intervention. Infant Behavior and Development, 34, 72-80. doi:10.1016/j.infbeh.2010.09.009

[36] Brett, J., Staniszewska, S., Newburn, M., Jones, N. and Taylor, L. (2011) A systematic mapping review of effective interventions for communicating with, supporting and providing information to parents of preterm infants. BMJ Open, 2, e000023. doi:10.1136/bmjopen-2010-000023

[37] Whitelaw, A. and Sleath, K. (1985) Myth of the marsupial mother: Home care of very low birth weight babies in Bogota, Colombia. Lancet, 25, 1206-1208. doi:10.1016/S0140-6736(85)92877-6

[38] Ghavane, S., Murki, S., Subramanian, S., Gaddam, P., Kandraju, H. and Thumalla, S. (2012) Kangaroo mother care in Kangaroo ward for improving the growth and breastfeeding outcomes when reaching term gestational age in very low birth weight infants. Acta Paediatrica, 101, e545-e549. doi:10.1111/apa.12023

[39] Field., T.M., Schanberg, S.M., Scafidi, F., Bauer, C.R., Vega-Lahr, N., Garcia, R., Nystrom, J. and Kuhn, C.M. (1986) Tactile/kinesthetic stimulation effects on preterm neonates. Pediatrics, 77, 654-658.

[40] Ruiz-Peláez, J.G., Charpak, N. and Cuervo, L.G. (2004) Kangaroo mother care, an example to follow from developing countries. British Medical Journal, 329, 1179-1181. doi:10.1136/bmj.329.7475.1179

[41] Ahn, H.Y., Lee, J. and Shin, H.J. (2010) Kangaroo care on premature infant growth and maternal attachment and post-partum depression in South Korea. Journal of Tropi- cal Pediatrics, 56, 342-344. doi:10.1093/tropej/fmq063

[42] Tessier, R., Charpak, N., Giron, M., Cristo, M., de Calume, Z.F. and Ruiz-Peláez, J.G. (2009) Kangaroo mother care, home environment and father involvement in the first year of life: A randomized controlled study. Acta $\mathrm{Pa}$ ediatrica, 98, 1444-1450. doi:10.1111/j.1651-2227.2009.01370.x

[43] Johnson, A.N. (2007) The maternal experience of kangaroo holding. Journal of Obstetric, Gynecologic, and Neonatal Nursing, 36, 568-573. doi:10.1111/j.1552-6909.2007.00187.x

[44] de Macedo, E.C., Cruvinel, F., Lukasova, K. and D’Antino, M.E. (2007) The mood variation in mothers of preterm infants in Kangaroo mother care and conventional incubator care. Journal of Tropical Pediatrics, 53, 344346. doi:10.1093/tropej/fmm076

[45] Cong, X., Cusson, R.M., Hussain, N., Zhang, D. and Kelly, S.P. (2012) Kangaroo care and behavioral and physiologic pain responses in very-low-birth-weight twins: A case study. Pain Management Nursing, 13, 127-138. doi:10.1016/j.pmn.2010.10.035

[46] Cong, X., Cusson, R.M., Walsh, S., Hussain, N., Ludington-Hoe, S.M. and Zhang. D. (2012) Effects of skin-toskin contact on autonomic pain responses in preterm infants. The Journal of Pain, 7, 636-645. doi:10.1016/j.jpain.2012.02.008

[47] Nimbalkar, S.M., Chaudhary, N.S., Gadhavi, K.V. and Phatak, A. (2013) Kangaroo mother care in reducing pain in preterm neonates on heel prick. The Indian Journal of Pediatrics, 80, 6-10. doi:10.1007/s12098-012-0760-6

[48] Hernandez-Reif, M., Diego, M. and Field, T. (2007) Preterm infants show reduced stress behaviors and activity after 5 days of massage therapy. Infant Behavior and Development, 30, 557-561.

doi:10.1016/j.infbeh.2007.04.002

[49] Field, T.M. (1998) Massage therapy effects. American Psychologist, 53, 1270-1281. doi:10.1037/0003-066X.53.12.1270

[50] Dudek-Schriber, L. (2004) Parental stress in the neonatal intensive care unit and the influence of parent and infant characteristics. American Journal of Occupational Therapy, 58, 509-520. doi:10.5014/ajot.58.5.509

[51] Ravn, I.H., Smith, L., Smeby, N.A., Kynoe, N.M., Sandvik, L., Bunch, E.H. and Lindemann, R. (2012) Effects of early mother-infant intervention on outcomes in mothers and moderately and late preterm infants at age 1 year: A randomized controlled trial. Infant Behavior and Development, 35, 36-47. doi:10.1016/j.infbeh.2011.09.006

[52] Kitamura, N., Nagahama, T., Ishizaki, Y., Kinoshita, Y. and Kaneko, K. (2009) Effects of interview on mood status of pregnant women with high-risk delivery. Pediatrics International, 51, 498-501. doi:10.1111/j.1442-200X.2008.02784.X 